\title{
Review of: "Risk factors for the severity of Guillain- Barré syndrome and predictors of short-term prognosis of severe Guillain-Barré syndrome"
}

\author{
Zuneng Lu
}

Potential competing interests: The author(s) declared that no potential competing interests exist.

Review of: "Risk factors for the severity of Guillain-Barré syndrome and predictors of short-term prognosis of severe Guillain-Barré syndrome"

Shuping $\mathrm{Liu}^{1}$, Zuneng $\mathrm{Lu}^{1 *}$

1 Department of Neurology, Renmin Hospital of Wuhan University, Wuhan, China

Correspondence to: Prof. Lu Zuneng, Department of Neurology, Renmin Hospital of Wuhan University, Wuhan 430060, Hubei Province, P.R. China (tel.: +86 13995672166; e-mail: Izn196480@126.com) As an immune-mediated acute peripheral neuropathy, Guillain-Barré syndrome (GBS) is heterogeneous in severity of neurological deficits and prognosis. Although intravenous immunoglobulin (IVIg) and plasma exchange are available and effective in majority of patients, 25 percent of patients progress to severe respiratory insufficiency, 20 percent remain severely disabled, and about 5 percent die ${ }^{1}$. In view of this, early identification of risk factors for poor prognosis and timely intervention are particularly important. Therefore, we really appreciate the study of risk factors for the severity of GBS and predictors of short-term prognosis of severe GBS². Nonetheless, issues on study design and analysis has several limitations. Firstly, as the authors demonstrated, variables that were significant in univariate analysis were further analyzed in multivariate regression analysis. But from a statistical point of view, multiple factor regression analysis requires a relatively larger sample size. For example, the authors choose the five variables, such as a recent surgical history, lower MRC scores on admission, lower MRC scores at nadir, treatment with $\mathrm{MV}$, and pneumonia complication, as the independent variables in multiple regression analysis when talking about the predictors associated with poor short-term progress, at least 50 patients with a poor prognosis were needed. Obviously, the sample size of patient with poor prognosis $(n=45)$ in this article was insufficient.

Secondly, the exact definition of mild or severe patients was not properly described. Patients with HFGS score $\geq 4$ points at nadir were classified into the severe GBS group, as the authors suggest, and then a total of 65 severe GBS patients were divided into groups either with poor progress (HFGS $\geq 3$ ) or good progress at discharge. The authors' definition of mild and severe patients inevitably leaving out the patients who peaked with milder symptoms $(\mathrm{HFGS}=3)$ but had a poorer prognosis, especially those elderly patients suffered from multiple complications, such as autonomic dysfunction and pneumonia, because some studies have indicated that the clinical course in mild patients may not be as mild as expected. Up to 
$38 \%$ of patients with a mild form (GBS disability score 1 or 2 ) of GBS-reported problems in hand function and running after 6 months follow-up despite the fact that $22 \%$ of them received treatment ${ }^{3}$.

Thirdly, with unable to walk independently at discharge as indicator of poor prognosis, deaths within recovery period would be underestimated. Studies have shown that fifteen (2.8\%) of 527 patients with GBS died within 6 months of follow-up at highly variable time points during the disease course, with a median time from onset of weakness to death of 76 days (interquartile range 23-152 days) and 67 percent of patients died during the recovery phase after neurologic improvement ${ }^{4}$. In this respect, the lack of followup made the conclusions less convincing.

Another concern is that, the subjects were excluded from the study if they were diagnosed with either bickerstaff encephalitis or Miller Fisher syndrome. We would like to know whether the authors included only typical GBS. If this is the case, electromyography (EMG) analysis is necessary, since studies have shown neurophysiological testing is helpful for assessing risk of respiratory failure, which is highest in patients with evidence of demyelination and very low in those without both $55.6 \%$ conduction block of the common peroneal nerve and a $20 \%$ reduction in vital capacity ${ }^{5}$.

\section{Acknowledgement}

This work was supported by grants from the Independent scientific research project of Wuhan University (2042020kf0053) and the National Natural Science Foundation of China (81971055). The sponsors played no role in the study design, data collection, and analysis, or decision to submit the article for publication.

\section{Disclosure Statement}

The authors declare no financial or other conflicts of interest.

\section{Reference}

1. van den Berg B, Walgaard C, Drenthen J, et al. Guillain-Barre syndrome: pathogenesis, diagnosis, treatment and prognosis. Nat Rev Neurol 2014; 10: 469-482. DOI: 10.1038/nrneurol.2014.121.

2. Puyuan Wen, Lisha Wang, Hong Liu, et al. Risk factors for the severity of Guillain-Barré syndrome and predictors of short-term prognosis of severe Guillain-Barré syndrome. Sci Rep 2021; 11:11578. DOI: 10.1038/s41598-021-91132-3.

3. Van Koningsveld R, Schmitz PI, Ang CW, et al. Infections and course of disease in mild forms of GuillainBarré syndrome. Neurology 2002;58:610-14.

4. Bianca van den Berg, Carina Bunschoten, Pieter A van Doorn, et al. Mortality in Guillain-Barre syndrome. Neurology 2013;80:1650-1654. DOI: 10.1212/WNL.0b013e3182904fcc.

5. Marie-Christine Durand, Raphaël Porcher, David Orlikowski, et al. Clinical and electrophysiological predictors of respiratory failure in Guillain-Barré syndrome: a prospective study. Lancet Neurol. 2006;5:1021-1028.DOI: 10.1016/S1474-4422(06)70603-2. 
